\title{
تطبيق النظرية السلوكية في تعليم مهارة الكلام بمعهد الرمة للبنات مالانج
}

\author{
(دراسة الحالة) \\ Ikke Wulandari \\ ikkewulandari@iainbengkulu.ac.id
}

\begin{abstract}
The purpose of this research is to: (1) to reveal the application of the behavioral theory in the teaching of speech skills in Boarding school Ar Rahmah Malang for Girls (2) to describe the results of teaching speech skills from the application of the behavioral theory in Boarding school Ar Rahmah Malang for Girls (3) to describe the disadvantages and advantages of the application of behavioral theory in teaching speech skills in Boarding school Ar Rahmah Malang for Girls

This type of qualitative research examines the case study method and obtains data from the interview, observation and documentation. The researcher chose data analysis techniques, such as the Miles and Huberman analysis of data classification, data presentation, and summary / conclusion of data. The researcher chose to examine the data on extension of participation and triangulation.

The results of the research: 1) The application of the activities of teaching the skill of speech in the behavioral theory at in Boarding school Ar Rahmah Malang for Girls, vary from two types is teaching the skill of speech in the classroom, including: Speak and memorize the Arabic language. And on the outside of them: giving the vocabulary, the conversation after maghrib, and test vocabulary of language, training speech, the implementation of learning activities of the skill of speech in Boarding school Ar Rahmah Malang for Girls in accordance with the theory of behavior. Because there are educational activities inside and outside the classroom, there is repetition, imitation, conservation and punishment. 2) and the results of teaching the skill of speech in the behavioral theory in Boarding school Ar Rahmah Malang for Girls in the classroom suitable for the promotion of the skill of speech. The researcher looked at the results of the conversation article 100\% success of 24 student. It is the article of the dialogue 93\% success of 30 student. 3) The advantages of applying the behavioral theory in the teaching of the skill of speech in Boarding school Ar Rahmah Malang for Girls suitable for the material of conversation or exchange that need to practice conservation and speak in Arabic. The disadvantages of the application of behavioral theory in the teaching of speech skill in Boarding school Ar Rahmah Malang for Girls there is not the language environment committee on the example language system, fewer applications do not use daily language, fewer penalties that do not use language.
\end{abstract}


التي يمكن توليدها من خلال مكافأة .وتؤكد هذه

النظرية أيضا إتقان الكلام في التدريس.

هذه النظرية السلوكية، كل السلوك البشري

يصبح السلوك اللغوي ويتم مظهر من مظاهر المثير اللغة والاستجابة بشكل مستمر يصبح عادة. وبناء على هذه النظرية، ويتم تعلم اللغة من خلال إعطاء الأولوية لإدخال مهارات الاستماع والكلام من المهارات الأخرى، وإعطاء تمارين واستخدام اللغة بشكل فعال ومستمر، وخلق بيئة اللغة الجيدة، واستخدام وسائل الإعلام التي تتيح للطلاب للاستماع والتفاعل مع الناطقين بها، التعود الدافع بحيث تصبح اللغة الأجنبية السلوك المعتاد. كانت النظرية السلوكية مناسبة لإكتساب قدرة التي تحتاج إلى ممارسة والتعود تحتوي على عناصر من سرعة العفوية والمرونة والمتانة. وعيوب هذه النظرية، التعليم يركز فقط إلى أستاذة بشكل ميكانيك حول الطالب ويركز إلى نتيجة .اعتبر التلاميذ السلبي، والطالب يستمعون فقط، يحفظ شرح المعلم أن المعلم المركزي والسلطوي.؛ ولكن في الواقع، وقد انتقدت النظرية السلوكية لأغما غالبا ما تكون غير قادرة على شرح مواقف تعليمية معقدة، لأن الكثير من المتغيرات أو الأمور المتعلقة بالتعليم والتعلم أو التي يمكن تحويلها

${ }^{4}$ Rosyida Wahab dan Ni'mah Mamluatul, Memahami Konsep Dasar Pembelajaran Bahasa Arab (Malang: Uin Maliki Press 2012), hlm 1.
التعلم هو محاولة لمعرفة .وهذا النشاط يؤدي

الطلاب يتعلمون من خلال بفعالية وكفاءة '.كما ذكر نبابا، أن معنى التعلم هو عملية نقل إلى الاسمية لإعطاء الدرس 'بتعليمية مفيدة من المفترض أن "عملية صنع أو التسبب آخرين للتعلم .ويمكن أن نستنتج أن تعلم (عملية التعلم) هو النشاط (جهد) على المعلمين المتعمد لتعديل (تنظيم) المكونات المختلفة للتعليم والتعلم التي يتم توجيهها لتحقيق الوجهة المحددة. - - المدة

لفهم الأنشطة وعملية التعلم وكذلك العوامل التي تعيق عملية سلسة التعلم، يمتاج المعلمون إلى فهم بعض من نظرية التعلم .فهم نظرية التعلم والتقدم الطلاب المفترضة .وبالإضافة إلى ذلك، مع مساعدة من نظريات ومفاهيم ومبادئ المعلمين تعلم أن إدارة التعلم بشكل أفضل.؟ وهناك عدة أنواع من نظريات التعلم، واحدة منها هي النظرية السلوكية .تفترض النظرية السلوكية أن اللغة تكتسب من خلال لغة عملية التكرار .انه يتقن من خلال المثير والرد الحركة . بحموعة هذه النظرية تشعر بالقلق مع افتتاح التعلم

${ }^{1} \square$ Muhaimin M.A. Dkk. Strategi Belajar Mengajar, (Surabaya: CV. Citra Media, 1996), hlm 99.

${ }^{2} \square$ Jos D Parera, Lingustik Edukasional, (Jakarta: Erlangga 1997), hlm 24-25

${ }^{3} \square$ Machmudah Umi dan Wahab Rosyidi Abdul. Active Learning Dalam Pembelajaran Bahasa Arab. (Malang: Uin Malang Press, 2008) hlm 12. 
التحفيظ، يقضون عقوبة في حالة واحدة، لا يظهر البنية النحوية على حدة، وأفضل مع نظام استناجي، ويركز على الكلام، واستخدام أكثر من لغة في الاتصالات والعديد استخدام مختبر اللغة، وتقدم مكافآت للاستجابة الإيجابية والدعم للغة، لاحظ اللغة ليس المحتوى اللغة.

\section{مفهوم النظرية الساوكية}

منطلق هذه النظرية، هو أن التعليم عملية

تأثر الإنسان بالحوادث المحيطة به التي تعطى التجارب والانفعالات. ' يقصد بالتعلم هو تغيير السلوك الذي يحدث بسبب وجود المثيرات والاستجابات الملحوظة، وتعتبر هذه النظرية أن الانسان قد تعلم ان استطاع أن يبرز تغيير السلوك. تركز هذه النظرية على ما يمكن النظر إليه وهو السلوك الموضوعي (Obyektif)، والواقعي ويمكن

‘(Empiris)

ملاحظته (Observable).

نظرية التعلم السلوكية هي نظرية التعلم التي

وضعت المزيد من التركيز على السلوك البشري . النظرية السلوكية يعتبر الفرد بأها رد الفعل قادرة على الاستجابة للبيئة .ووسوف خبرة صيانة تشكيل

${ }^{5} \square$ Djali, Psikologi Pendidikan, (Jakarta: Bumi Aksara, 2011)hal.75

${ }^{6} \square$ R.Moeslihatoen, Interaksi Belajar Mengajar, (Malang: FIP IKIP, 19891) hal 11
إلى علاقة المثيرة والاستجابة لها .هذه النظرية غير قادرة على تفسير الانحرافات التي تحدث العلاقة بين المثيرة والاستجابة لما. يميل النظرية السلوكية أيضا أن يؤدي الطلاب على التفكير الخطي ومتقاربة، غير البناء وغير منتجة .بآراء هذه النظرية أن التعلم هو عملية تشكيل أو تشكيل، الذي يجمع الطلاب داخل أو تحقيق أهداف معينة، مما يجعل الطلاب ليسوا أحرارا في أن تكون خلاقة ومبدعة .على الرغم من العديد من العوامل المؤثرة التي تؤثر على عملية التعلم .لذلك ليست بسيطة كما وصفت نظرية السلوكية. معهد الرحمة هي واحدة من المدارس المشهورة، المدرسة التي ملتزمة بتنفيذ مفهوم وممارسة التربية الإسلامية في جميع جوانبه في شكل التوحيد القائم على التعليم .التعليم باعتباره زرع الأدب هو عملية التعليم المشهورة لا يعلم العلم موجودة في الكتاب فقد، ولكن أيضا المواقف والإجراءات، والآداب، و والعطف والإخلاص واحد الجسدية والعقلية على استعداد لقبول هبة من الله سبحانه وتعالى .واحدة مزايا من الرحمة تستخدم لغتين ، وهي العربية والإنجليزية، وبيئة اللغوية.

في هذه الحالة، معهد الرحمة واحدة التي تستخدم نظرية السلوكية في تعلم مهارة الكالام. انطلاقا من خصائص نظرية وهذا يعني، عرض المواد مع مزيد من الحوار، والمزيد من التقاليد والتعبير 
تدعم هذه السلوكات بممارسات أو تدريبات مرتبطة لتحويلها إلى السلكات دائمة نسبيًا.

\section{تطبيق النظرية السلوكية في تعليم اللغة العربية}

تطبيق تعليم اللغة العربية في ضوء النظرية

السلوكية إن المعلم هو السند الحقيقي والركن

الأساسي، لذالك يحتل المكانة المرموقة في العملية التعليمية، ومحورها، ويتمثل دورة في الهيئة بيئة التعلم لتشجيع الطلاب لتعلم السلوك المرغوب، يعمل على تعزيزة. إذن فكل مايقوم به المعلم إزاء التلميذ ويوجه إليه مثيرا، فالسؤال الذي يطرحهى المعلم، والصورة التي يظهرها على اللوح تمثل المثيرات لأهفا تدفع التلميذ للتكلم ، لذلك فإجابة التلميذ على السؤال وتعليقه على الصورة تعد استجابات، تتعليق المعلم على تلك الإجابات تعد تعزيزا أو سلبيا. فإذا الإجاباتصحيحة يعمد المعلم إلى تدعيمها والتعقيب عليها بالقبول والشكر والتأييد ، إذا كانت الخطيئة يعمل المعلم على توقيفها أو إزالتها، وذلك هو التعزيز السبي. '1. أما خطوات التعليم على ضوء النظرية

$$
\text { السلوكية، فكما يلي: 'أ) تحديد أهداف التعليم }
$$

'عبد المجيد عيساني، نظريات التعلم وتطبيقها في

علوم اللغة: اكتساب المهارات اللغوية، القاهرة: دار الكتاب والحديث،

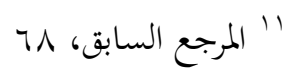

سلوكهم ' .نظرية السلوكية هي نظرية التنمية السلوكية، والتي يمكن قياسها، لاحظ وأنتج من قبل الطلاب في الاستجابة للمؤثرات ـويمكن تعزيز الاستجابة للمؤثرات عن طريق ردود فعل إيجابية أو سلبية على الأوضاع السلوك المطلوب .يستخدم العقاب أحيانا للقضاء أو الحد من أعمال غير صحيح، تليها واصفا الإجراء المطلوب`. وبتحليل هذا التعريف السلوكي للتعلم، بتح أنه يتكون من العناصر الأتية:

1. إنسان يمثل كائناً عضويًا حيًا (المتعلم) Y. بيئية تعلمية - مثيرات أو خبرات r. تفاعل الي بين الإنسان والخبرات والمثيرات

ع. استجابة المتعلم للمثيرات وحدوث ارتباطات

$$
\text { ه. تعزيز وممارسات وتدريب }
$$

أي أن التعلم في النظرية السلوكية يتطلب شروطا موضوعية وهي : عضوبي (الإنسان) والبيئة التعلمية (المثيرات) وتفاعل الإنسان المتعلن مع المثيرات، وقيامه باستجابات متلائمة وحدوث ارتباطات، ومن ثم تتوج السلوكات المكتسبة، بتعزيز من الخارج

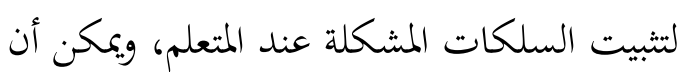

${ }^{7}$ Machmudah Umi dan Wahab Rosyidi Abdul. Active Learning Dalam Pembelajaran Bahasa

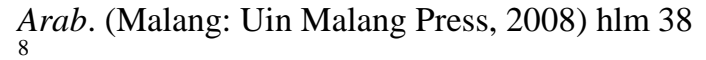

هُمُّجّ محمود الخوالدة، أساس بناء المناهج التربوية وتصميم

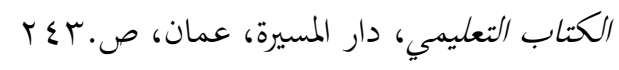


1) شيوع استعمال للهجات العامية إلى الحدد الذي جعلها تضايق الفصحى، وتحاصرها في بجالات ضيقة جدا ، فالعامية هي لغة التواصل في البيت والمجتمع، وساحة المدرسة، وكثيرا ما تمتد إلى لغة الدراسة، فالكثير من المدرسين يدرسون موادهم باللغة العامية، ولا نعدو الصواب لو قلنا إنها تسربت إلى درس اللغة العربية نفسها. Y) عدم اهتمام مدرسي المواد الأخرى باللغة العربة ، فاقتصار الاهتمام بها على مدرس اللغة العربية وبها أن اللغة اللغة تكتسب بالسماع والممارسة فإن ذلك يقتضي أن يحاط المتعلم ببيئة لا يسمع فيها لحنا، ولاشططا عن الفصيحة، لذالك ففي مثل هذه الحال ينطبق على تعليم العربية

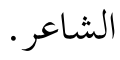

r) من المشكلات تعليم اللغة العربية أن مناهجها لا تؤسس لإعداد قارئ جيد، فالقارئ في العربية بطيء جدا كثير

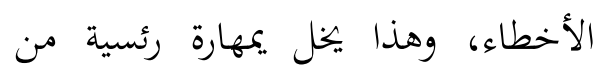
مهارات اللغة يتأسس عليها اكتساب المعلومات في الميادين المختلفة، ويمتد الخلل فيها المعلومات التي يراد اكتساهما بالقراءة.

ب) تحليل البيئة التعليمية الواقعية ومعرفة المعلومات السابقة

$$
\begin{aligned}
& \text { للمتعلمين } \\
& \text { ت) تعيين المواد التعليمية } \\
& \text { ث) تقسيم المواد التعليميةإلى الجزئيات } \\
& \text { ج) عرض المواد التعليمية }
\end{aligned}
$$

ح) إعطاء المثيرات (Stimulus) عن الطريق السؤال والجواب شفهيا أو تجربيا أو عن الطريق الواجبات. خ) ملاحضة ودراسة استجابات المتعلمين (respon) د) إجراء عملية التعليم. مشكلات في التعلم اللغة العربية مع ما للعربية من ميزات تقدمت بها على لى لماني

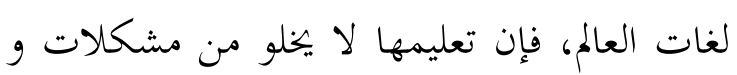
صعوبات يجب وضع اليد عليها ووضع المعالجات الممكنة لها في مجال التعليم. إن مشكلات التعليم العربية تتوزع بين مجالين. أولا مشكلات عامة، وأخرى خاصة وسنقتصر هنا على عرض المشكلات العامة أما الخاصة التي تتصل بمهارات اللغة فسنتعرض لها عند الحديث عن تعليم تلك المهارات في الفصول اللاحقة، ومن مشكلات العامة ما يأتي:

$$
\text { وتعليمها. (بغداد: دار المناهج للنشر والتوزيع) ص علية، مهارات الاتصال اللغوي }
$$


بحاجتهم إلى اللغة العربية في دراسة المواد

$$
\text { الإخرى. }
$$

ندرة المواقف التي يمارس فيه الطلبة

$$
\text { مهارات اللغة، ويتدربون عليها. }
$$

شيوع الأخطاء اللغوية، والإملائية

$$
\text { في وسائل الإعلام المختلفة. }
$$

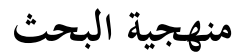

مدخل البحث و منهجه البحت

إستخدمت الباحثة في هذا البحث المدخل

الكيفي والمنهج باستخدام شكل دراسة الحالة. لأن أرادت الباحثة أن تركز على فهم وصف الظاهرة

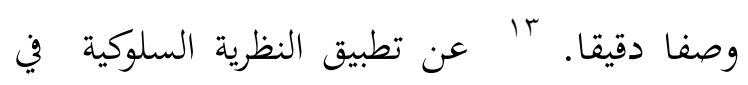
تعليم مهارة الكلام بععهد الرحمة للبنات مالانج. وحاولت الباحثة عن معرفة بيانات البحث للوصول إلى نتائج البحث. دراسة الحالة هي دراسة متعمقة للحدث، والبيئة، وبعض الحالات التي تسمح صريحة 'أو فهم شيئاء'.

أرادت الباحثة في هذا البحث أن توضيح عن تطبيق النظرية السلوكية في تعليم مهارة الكلام بمعهد الرحمة للبنات مالانج ولمعرفة مشكلات تطبيق النظرية السلوكية في تعليم مهارة الكلام بمعهد الرحمة

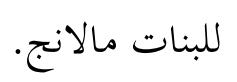

${ }^{14}$ Lexy J. Moleong, Metodologi Penelitian Kualitatif, (Bandung; PT Remaja Rosdakarya, 2006), hlm. 3

ع) جفاف الكثير من القواعد النحوية واعتمادها على العرف الذي يخالف في

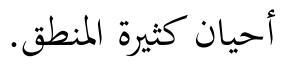
ه) عدم تأهيل معلمي اللغة العربية لاستخدام تقنيات التعليم الحديث في تعليم اللغة العربية مما أفقد درس اللغة العربية مما حصل من تطور في طرائق التعليم وتكنولوجيا الاتصال التي دخلت ميدان

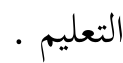
7) إناطة تعليم اللغة العربية يمعلمين ومدرسين غير مؤهلين تربويا لتعليم اللغة العربية فقد نجد الكثير ممن يتولون تعليم اللغة العربية

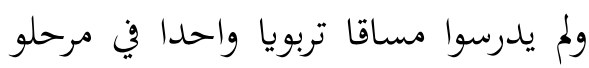
الإعداد خريبو كليات الأدب. V حاجة اللغة العربية المكتوبة إلى الشكل،

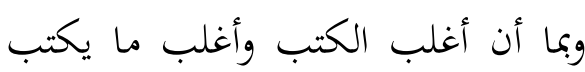
خال من شكل فإن ذلك بعد مشكلة للقارئ غير المؤهل في مجال النحو والصرف. ᄉ) ارتباط المعاني بالحركات الإعرابية فلو. 9) التعامل مع الفروع اللغة العربية باعتبارها مواد منفصلة عن بعضها يؤدي إلى تشتت القدرة اللغوية لدى المتعلم. عدم ربط اللغة العربية بالمواد الدراسية الأخرى، عدم إشعار الطلبة 
تعليم مهارة الكلام. وتحدد الباحثة مصدر البيانات عن طريق تحديد الاعتبارات وأغراض محددة.

\section{1 - الماحضة}

طريقة الملاحظة هي وسيلة لجمع

البيانات باستخدام الحواس، وخاصة حاسة

البصر والسمع .الملاحظة نفسها يمكن أن

تفسر تسجيل منهجي وجرى التحقيق

ملاحظة الأعراض.

واستخدمت طريقة الملاحظة

للحصول على بيانات شاملة عن حالة

البيئة، الوسايل والمرافق المملوكة والمشكلات

التعلم المهارة الكلام في معهد الرحمة.

- لاحظت الباحثة عن كيفية تعليم

مهارة الكاملام لمرحلة الثانوية،

- لاحظت الباحثة عن مشكلات

في تعليم اللغة العربية خصوصا

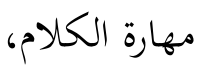

- لاحظت الباحثة عن الوسايل

الذي يستخدم في تعليم مهارة

الكلام داخل و خارج الفصل.

- - لاحظت الباحثة عن عن الأنشطة

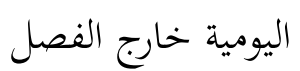

${ }^{15} \square$ Lexy J. Moleong, metodolgi Penelitian Kualitatif, (Bandung : PT Remaja Rosdakarya. 2007 ) hlm 127

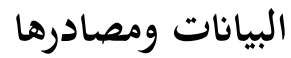

أما مصادر البيانات من هذا البحث كما

التالي: أخذت الباحثة البيانات من أفراد معينة من

المدرسة منهم :

(1) معلمة اللغة العربية للفصل

الأول و والثاني بمعهد الرحة

ل لبنات مالانج،

r) قسم اللغة بمعهد الرحمة للبنات

$$
\text { مالانج }
$$

r) والطلبة الفصل الأول والثاني

ع ) الوثائق المتعلقة بتعليم مهارة

الكالام داخل وخارج الفصل

مثل نتائج مهارة الكلام،

الجدوال اليومية الطلبة وغيرها.

إستخدمت الباحثة مصدرين: المصادر

الأساسية منها المصادر عند المقابلة بمعلمة اللغة

العربية، قسم اللغة ،الطلبة، وملاخظة عملية التعليم

مهارة الكلام داخل وخارج الفصل. أما المصادر الثانوية هو الوثائق المتعلقة عن تعليم اللغة العربية

$$
\text { داخل وخارج الفصل. }
$$

بدأت الباحثة باتصال والملاحظة والمقابلة

والوثائق مع الناس الذين لديهم معرفة عن تعليم اللغة العربية ، وخاصة عن استخدام النظرية السلوكية في 
تم تحضيرها وتتضمن على التمهيد أي

مرحلة التفاعل، وتقديم مادة التعليم.

r) مشكلات افي تعليم اللغة العربية بمعهد

الرحمة للبنات مالانج

r. الوثائق هي المصدار البيانات في كثير من

الأحيان موقعا هاما في البحث الكيفي

حتى يكون البحث كاملا. ^ا' الوثائق هي

طريقة لإعداد الوثائق باستخدام دليل صارم

لتسجيل مصادر معلومات محددة عن مقال

/المادة، والوصايا، والكتب، والقوانين،

وهلم جرا .في الشعور العام وثائق هي

البحث، التحقيق وبمع وحفظ وحيازة

$$
\text { واستخدام وتقديم وثائق } 19 .
$$

إستحدمت الباحثة هذه الوسيلة

لجمع بعض المعلومات عن كيفية تعليم

مهارة الكلام وهي طريقة البحث لطلب

الحقائق و والبيانات من الوثائق والكتب

والمجلات والرسائل والمذكورات وغيرها من

$$
\text { المواد لتعليم اللغة العربية. }
$$

وجدت الباحثة باستخدام الوثائق

${ }^{18}$ Muhammad Tholchah Hasan, Metode Peneltian Kualitatif Tinjauan Teoritis dan Praktis.(Surabaya: Visipress Media) 2011, hlm. 135

${ }^{19} \square$ Van Hoeve; Hassan Shadily. Ensiklopedia Indonesia, Jilid 7. (Jakarta: Ichtiar Baru). hlm 849.

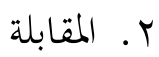

مقابلة هي الطريقة المستخدمة

للحصول على البيانات على شكل شفوي

كتابي من حوار أجراه المقابلة للحصول

على معلومات أو معرفة من مقابلتهم. '17.

وقد استخدم المقابلة عن تقنيات

جمع البيانات إذا أرادت الباحثة إلى إجراء

دراسة أولية للعثور على المشاكل التي يجب

التحقيق فيها، وإذا أرادت الباحثة إلى معرفة

الأشياء من المستطلعين أعمق ل' .ويمكن

إجراء مقابلات في منظم أو غير منظم،

ويمكن أن يتم من خلال وجها لوجه أو عبر

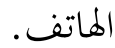

تستخدم الباحثة هذا الأسلوب لطلب

البيانات الى المدرسة إسمها أستاذة ميتى، و

بعض الطلبة.ولطلب البيانات محا يلي:

() عملية التعليم من حيث كتابة المعلمة

تحضير الدرس وتحديد الأهداف

والطرائق، والوسائل، وتحديد أساليب

التقويم.

r) تخطيط عملية التدريس من حيث

تطبيق أساليب التدريس وإجراءاته التي

${ }^{16} \square$ Hadi Sutrisno, Metodologi Research II. (Yogyakarta : Yayasan Penerbit Fak. Psikologi UGM. 1993) hlm 145

${ }^{17} \square$. Sugiyono, Metode Penelitian Pendidikan Pendekatan Kuantitatif, Kualitatif dan RnD, (Alfabeta Bandung) 2014 hlm. 33 ฯ 
1. تطبيق نشاطات تعليم مهارة الكلام

بالنظرية السلوكية في معهد الرحمة للبنات

مالانج تتنوع من نوعين هي تعليم مهارة

الكالام داخل الفصل منها: تكلم وحفظ

مادة اللغة العربية. وخارجها منها: إعطاء

المفرذات، المحادثة بعد المغريب، وإختبار

المفرذات اللغة ، تدريب الخطاب، تطبيق

نشاطات تعليم مهارة الكلام بمعهد الرحمة

للبنات مطابقا بالنظرية السلوكية ـ لأن

تجري نشاطات تعليم داخل الفصل

وخارخه يوجد التكرار والتقليد والحفظ

$$
\text { والعقاب. }
$$

ץ. نتائج تعليم مهارة الكلام بالنظرية

السلوكية في معهد الرحمة للبنات مالانج

داخل الفصل مناسب لترقية مهارة

الكالام. نظرت الباحثة نتائج من المادة

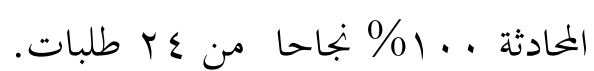

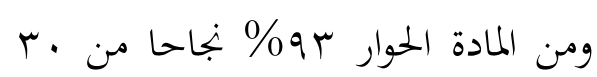

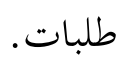

\section{المراجع}

عبد المجيد عيساني، نظريات التعلم وتطبيقها في

علوم اللغة: اكتساب المهارات اللغوية، لعابة

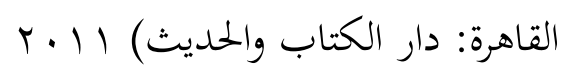

رشدي أحمد طعيمة، المرجع في تعليم اللغة العببية

للناطقين بلغات أخرى (الجزء الأول)،

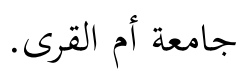

- - الصوار حالة من الأنشطة

التعليمية في المادة المحادثة والصرف

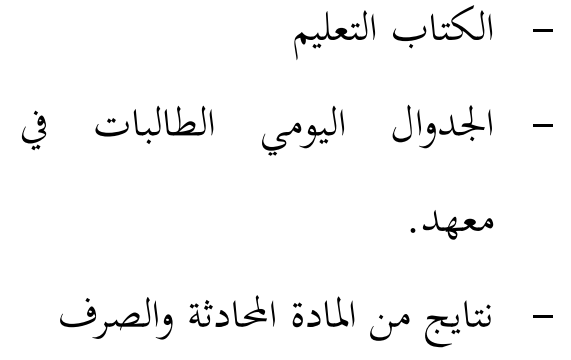

تحليل البيانات

تحليل البيانات في البحوث النوعية قامت

منذ ما قبل دخول الميدان، خلال الميدان، وبعد الانتهاء من هذا المجال .في هذه الحالة، وقال ناسوتيون، "بدأ التحليل وضعت منذ وشرح المشكلة، قبل أن تنقلب رحابة، وتستمر حتى لحظة إعداد نتائج البحوث .ومع ذلك، في البحث النوعي، وتحليل البيانات هو أكثر تركيزا أثناء عملية جنبا إلى جنب مع جمع البيانات الميدانية. ‘r بدأت تحليل البيانات الكيفية من الدخول إلى حقل الميدان أو بداية جمع البيانات حتى الخروج منه أو ليدات بعد إنتهاء من الجمع البيانات. ميلز وهوبرمان المكتوب في كتاب سغيونو يشير إلى أن تحليل البيانات الكيفية يتم بشكل التفاعل

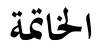

واختتاما في هذا البحث هو الاستنتاج كما

يلي:

${ }^{20} \square$ Sugiyono, Metode Penelitian Pendidikan Pendekatan Kuantitatif, Kualitatif dan RnD, (Alfabeta Bandung) 2014 hlm. 33 ฯ 
Hadi Sutrisno, Metodologi Research II.

(Yogyakarta : Yayasan Penerbit Fak. Psikologi UGM. 1993)

Jos D Parera, Lingustik Edukasional, (Jakarta: Erlangga ) 1997

Lexy J. Moleong, metodolgi Penelitian Kualitatif, (Bandung : PT Remaja Rosdakarya. 2007)

Muhaimin M.A. Dkk. Strategi Belajar Mengajar (Surabaya: CV. Citra

Machmudah Umi dan Wahab Rosyidi Abdul. Active Learning Dalam Pembelajaran Bahasa Arab. (Malang: Uin Malang Press )2008

R.Moeslihatoen, Interaksi Belajar Mengajar, (Malang: FIP IKIP) 19891

Sugiyono, Metode Penelitian Pendidikan Pendekatan Kuantitatif, Kualitatif dan RnD, (Alfabeta Bandung) 2014

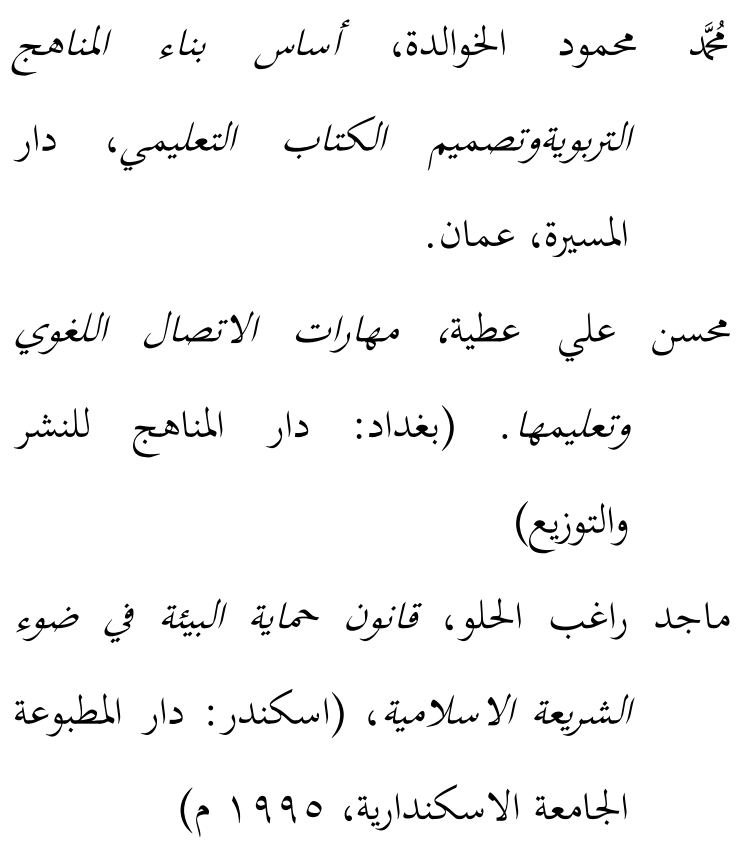

Aziz Fachrurrazi dan Erta Mahyudin, Pembelajaran Bahasa Asing: Metode Tradisional dan Kontemporer, (Jakarta: Bania Publishing $) 2010$ Djali,Psikologi Pendidikan,

(Jakarta: Bumi Aksara) 2011 